\title{
Verification of a Novel Approach to Predicting Effects of Antibiotic Combinations: In Vitro Dynamic Model Study with Daptomycin and Gentamicin against Staphylococcus aureus
}

\author{
Maria V. Golikova ${ }^{1, * \mathbb{D}}$, Elena N. Strukova ${ }^{1}$, Yury A. Portnoy ${ }^{1}$, Stephen H. Zinner ${ }^{2}$ and \\ Alexander A. Firsov ${ }^{1,+}$ \\ 1 Department of Pharmacokinetics \& Pharmacodynamics, Gause Institute of New Antibiotics, 11 Bolshaya \\ Pirogovskaya Street, 119021 Moscow, Russia; lena-stru@inbox.ru (E.N.S.); yaportnoy@gmail.com (Y.A.P.); \\ kindyn@gmail.com (A.A.F.) \\ 2 Department of Medicine, Harvard Medical School and Mount Auburn Hospital, 330 Mount Auburn St., \\ Cambridge, MA 02138, USA; szinner@mah.harvard.edu \\ * Correspondence: golikovaka@gmail.com; Tel.: +7-926-7077198 \\ + This author has passed away.
}

Received: 29 July 2020; Accepted: 24 August 2020; Published: 25 August 2020

\begin{abstract}
To explore whether susceptibility testing with antibiotic combinations at pharmacokinetically derived concentration ratios is predictive of the antimicrobial effect, a Staphylococcus aureus strain was exposed to daptomycin and gentamicin alone or in combination in multiple dosing experiments. The susceptibility of the S. aureus strain to daptomycin and gentamicin in combination was tested at concentration ratios equal to the ratios of $24 \mathrm{~h}$ areas under the concentration-time curve $\left(\mathrm{AUC}_{24} \mathrm{~s}\right)$ of antibiotics simulated in an in vitro dynamic model in five-day treatments. The MICs of daptomycin and gentamicin decreased in the presence of each other; this led to an increase in the antibiotic $\mathrm{AUC}_{24} / \mathrm{MIC}$ ratios and the antibacterial effects. Effects of single and combined treatments were plotted against the $\mathrm{AUC}_{24} / \mathrm{MIC}$ ratios of daptomycin or gentamicin, and a significant sigmoid relationship was obtained. Similarly, when the effects of single and combined treatments were related to the total exposure of both drugs (the sum of $\mathrm{AUC}_{24} / \mathrm{MIC}$ ratios $\left.\left(\sum \mathrm{AUC}_{24} / \mathrm{MIC}\right)\right)$, a significant sigmoid relationship was obtained. These findings suggest that (1) the effects of antibiotic combinations can be predicted by $\mathrm{AUC}_{24} / \mathrm{MICs}$ using MICs of each antibacterial determined at pharmacokinetically derived concentration ratios; (2) $\sum A U C_{24} / \mathrm{MIC}$ is a reliable predictor of the antibacterial effects of antibiotic combinations.
\end{abstract}

Keywords: daptomycin-gentamicin combination; in vitro model; anti-staphylococcal effect

\section{Introduction}

The extensive use of daptomycin in clinical practice has led to decreased daptomycin effectiveness due to the emergence of bacterial resistance [1-15]. Daptomycin treatment failures have been reported in patients with Staphylococcus aureus infections after prolonged daptomycin exposure [1-8] and also following vancomycin administration that was associated with vancomycin-resistant S. aureus [9-15]. Similarly, infections caused by vancomycin-resistant enterococci with lowered susceptibility to daptomycin can be difficult to treat even at high dosing regimens of daptomycin [16]. Combination antibiotic therapy can be a valuable option to improve the antibacterial effectiveness of daptomycin. In our previous study, the combination of daptomycin with rifampin used at subtherapeutic doses was characterized by an increased anti-staphylococcal effect and 
the pharmacodynamic interaction was interpreted as synergistic [17]. The enhanced efficiency of daptomycin-rifampicin combinations was predicted by the ratio of the 24-area under the concentration-time curve $\left(\mathrm{AUC}_{24}\right)$ to the MIC of daptomycin determined in the presence of rifampin. Susceptibility testing of $S$. aureus to daptomycin or rifampin in combination was performed at pharmacokinetically derived antibiotic concentration ratios, which were strongly related to the daptomycin-to-rifampicin $\mathrm{AUC}_{24}$ ratios used in subsequent pharmacokinetic simulations.

Verification of this approach with daptomycin in combination with other anti-staphylococcal antibiotics such as gentamicin is of interest. The synergistic interaction between daptomycin (as a cell membrane targeting antibacterial effects) and gentamicin (ribosomal targeting) can be hypothesized based on existing knowledge that daptomycin acts on bacterial cell membrane and increases its envelope permeability to another drug such as gentamicin (uptake effect) [18]. However, some previously conducted studies exploring the synergistic potential of daptomycin-gentamicin combinations [19-27] yielded controversial results. In some checkerboard studies with staphylococci, daptomycin plus gentamicin combinations were mostly synergistic [19], while in others, they were additive or indifferent $[20,21]$. In static time-kill experiments with these drugs, synergy was reported as prevalent [22,23]. Similar discrepancies were observed in studies using in vitro dynamic models that consider differences in daptomycin and gentamicin pharmacokinetics [24-27]. The effects of therapeutic exposures of daptomycin-gentamicin combinations were either enhanced or improved [24-26] but were antagonistic in one study [27]. Moreover, the in vivo anti-staphylococcal efficiency of daptomycin was not enhanced by the addition of gentamicin during treatment of experimental endocarditis in humanized rabbits [23].

To explore whether susceptibility testing with antibiotic combinations at pharmacokinetically derived concentration ratios is predictive of the antimicrobial effect, a clinical S. aureus strain was exposed to daptomycin and gentamicin in an in vitro dynamic model that simulates single and combined treatments. As therapeutic daptomycin exposures were characterized by a pronounced effect against staphylococci [28], subtherapeutic doses, which provide low antibacterial activity, were used in the present study. In contrast, gentamicin pharmacokinetics were simulated at therapeutic exposures, given previous in vitro studies that reported low $[25,26]$ or moderate $[29,30]$ anti-staphylococcal efficacy even at high peak concentrations at extended dosing interval regimens.

\section{Results}

\subsection{MICs of Daptomycin and Gentamicin Alone and in Combination}

Daptomycin-to-gentamicin concentration ratios used in MIC determinations corresponded to $\mathrm{AUC}_{24}$ ratios used in the pharmacodynamic simulations. The MICs of daptomycin and gentamicin alone and in combination are listed in Table 1. As follows from the table, the MIC of daptomycin in the presence of gentamicin decreased 4- to 16-fold when the aminoglycoside portion in the combination prevailed (daptomycin-to-gentamicin concentration ratios of 1:1.5, 1:2 and 1:5). When the daptomycin portion in the combination prevailed (1.5:1), daptomycin MIC decreased only two-fold compared to the MIC determined in the absence of gentamicin. At the same time, gentamicin MICs under the influence of daptomycin decreased 1.5- to 3-fold depending on the antibiotic concentration ratios. Changes in $S$. aureus susceptibility to daptomycin and gentamicin in the presence of each other were reflected in fractional inhibitory concentration (FIC) analysis (Table 1). As seen in the table, with increasing gentamicin portions in the combination from 0.67 (ratio 1.5:1) to 1.5 (ratio 1:1.5), the total (summation) fractional inhibitory concentration index ( $\sum F I C$ ) fell from 1.18 to 0.48 , revealing a change in the type of antibiotic interaction from indifferent to synergistic. Subsequent increases in the relative portion of gentamicin in the combination led to systematic increases of $\sum F I C$ index from 0.48 to 0.64 and from 0.64 to 0.70 at concentration ratios $1: 2$ and 1:5, respectively, reversing to an indifferent interaction. Thus, the lower EFIC showing synergy was associated with the proportion of antibiotics in the combination with relatively higher gentamicin concentrations. 
Table 1. MICs of daptomycin and gentamicin alone or in combination against Staphylococcus aureus 293 and respective FIC indices.

\begin{tabular}{ccccccc}
\hline \multirow{2}{*}{$\begin{array}{c}\text { Dosing } \\
\text { Regimen }\end{array}$} & $\begin{array}{c}\text { Daptomycin-to-Gentamicin } \\
\text { AUC24 Ratio }\end{array}$ & \multicolumn{2}{c}{ MIC (mg/L) } & \multicolumn{3}{c}{$\boldsymbol{F I C}$} \\
\cline { 3 - 7 } & - & 0.5 & - & - & - & - \\
\hline D30 & - & - & & - & - & - \\
D100 & - & - & 0.25 & - & - & - \\
\hline G65 & - & 0.25 & 0.17 & 0.5 & 0.68 & 1.18 \\
G160 & $1.5: 1$ & 0.06 & 0.09 & 0.12 & 0.36 & 0.48 \\
\hline D100 + G65 & $1: 1.5$ & 0.06 & 0.13 & 0.12 & 0.52 & 0.64 \\
D100 + G160 & $1: 2$ & 0.03 & 0.16 & 0.06 & 0.64 & 0.70 \\
D30 + G65 & $1: 5$ & & & \\
D30 + G160 & & & &
\end{tabular}

\subsection{Antibiotic Pharmacodynamics with S. aureus}

Time-kill kinetics of S. aureus 293 exposed to daptomycin and gentamicin alone or in combination in the in vitro dynamic model are shown in Figure 1 . As seen in the figure, single daptomycin treatments at $\mathrm{AUC}_{24} 30$ or $100 \mathrm{mg} \cdot \mathrm{h} / \mathrm{L}$ were associated with only a slight anti-staphylococcal effect within the first 12 or $24 \mathrm{~h}$ from the beginning of the experiment, respectively, followed by regrowth to cell density that exceeded the initial numbers. Gentamicin produced more pronounced anti-staphylococcal effects than daptomycin and within the first $24 \mathrm{~h}$ of treatment reduced initial cell numbers to either $4 \log \mathrm{CFU} / \mathrm{mL}\left(\mathrm{AUC}_{24} 65 \mathrm{mg} \cdot \mathrm{h} / \mathrm{L}\right.$ ) or below the limit of detection $\left(\mathrm{AUC}_{24} 160 \mathrm{mg} \cdot \mathrm{h} / \mathrm{L}\right)$. However, rapid initial killing by gentamicin was followed by fast regrowth and the numbers of surviving organisms at the end of the treatment were equal or close to the initial inocula (8 $\log \mathrm{CFU} / \mathrm{mL}$ at $\mathrm{AUC}_{24} 65 \mathrm{mg} \cdot \mathrm{h} / \mathrm{L}$ or $7 \log \mathrm{CFU} / \mathrm{mL}$ at $\mathrm{AUC}_{24} 160 \mathrm{mg} \cdot \mathrm{h} / \mathrm{L}$ ). However, all combined treatments with daptomycin and gentamicin were associated with more pronounced antibacterial effects than respective mono-treatments. Comparing the numbers of surviving staphylococci in treatments with the lower dose of gentamicin alone (as the more active single agent) and in combined treatments (regimens G65 versus D30+G65 and D100+G65), independent of the relative amount of daptomycin, the antibiotic combinations produced more pronounced initial bacterial killing (reduction to the limit of detection) and weaker regrowth (Figure 1a,c). At the higher gentamicin $\mathrm{AUC}_{24}(\mathrm{G} 160)$, the initial killing of $S$ aureus was the same in mono- and in combined treatments; cell numbers reached the limit of detection during the first $24 \mathrm{~h}$. However, the regrowth phase was delayed in combined treatments and at the end of the experiment cell density reached $5.5 \log \mathrm{CFU} / \mathrm{mL}$ (ratio 1:5, regimen D30+G160) versus $7 \log \mathrm{CFU} / \mathrm{mL}$ with gentamicin alone (G160); bacterial regrowth was fully suppressed with regimen D100+G160 (ratio 1:1.5) (Figure 1b,d).

\subsection{Pharmacodynamic Bliss Independence-Based Drug Interaction Analysis}

To accurately assess the advantages of combined therapy, Bliss independence-based drug interaction analysis was performed using data obtained in the pharmacodynamic experiments (Figure 2). In all combination regimens, $E_{\mathrm{DG}}$ was significantly higher than $E_{\mathrm{IND}}$ and the respective lower limits of $95 \% \mathrm{CI}$ always were positive. Depending on the combination regimen, $\Delta E$ varied from 8 to $22 \%$, with the maximum $\Delta E$ value in regimen D100+G160 (1:1.5 $\mathrm{AUC}_{24}$ ratio). 


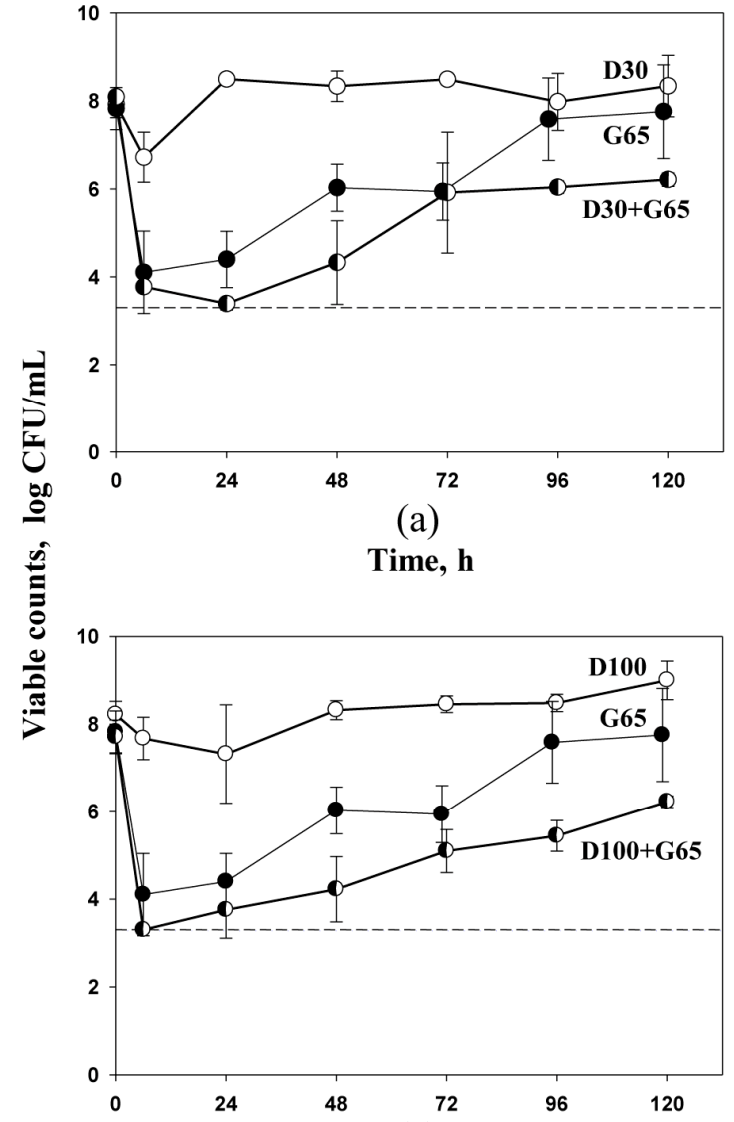

(c)

Time, h

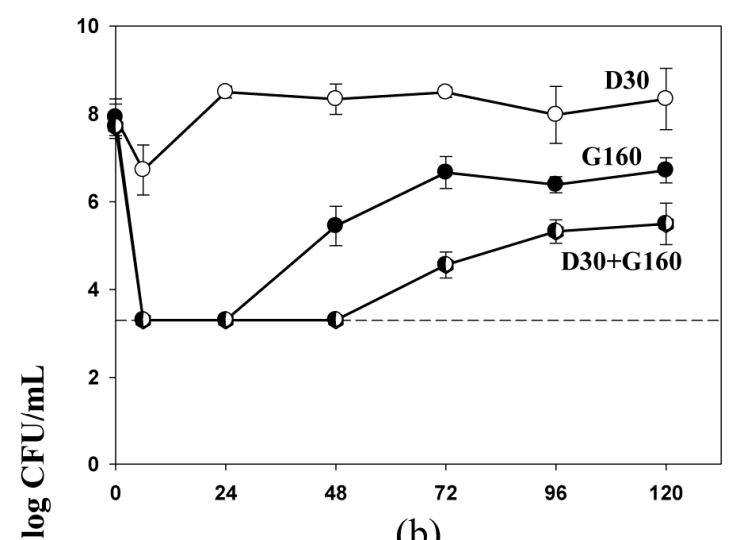

(b)

Time, h

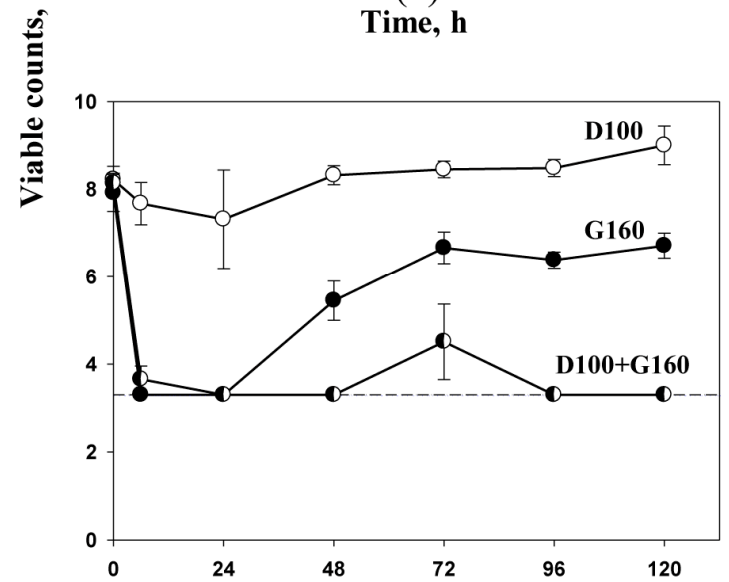

(d)

Figure 1. Time-kill curves of daptomycin and gentamicin, alone and in combination against S. aureus 293. Dosing regimens are indicated at each curve. Dotted lines indicate the limit of detection. Data are presented as arithmetic means \pm standard deviations. (a)-regimens D30, G65 and D30+G65; (b)—regimens D30, G160 and D30+G160; (c)—regimens D100, G65 and D100+G65; (d)—regimens D100, G 160 and D100+G160.

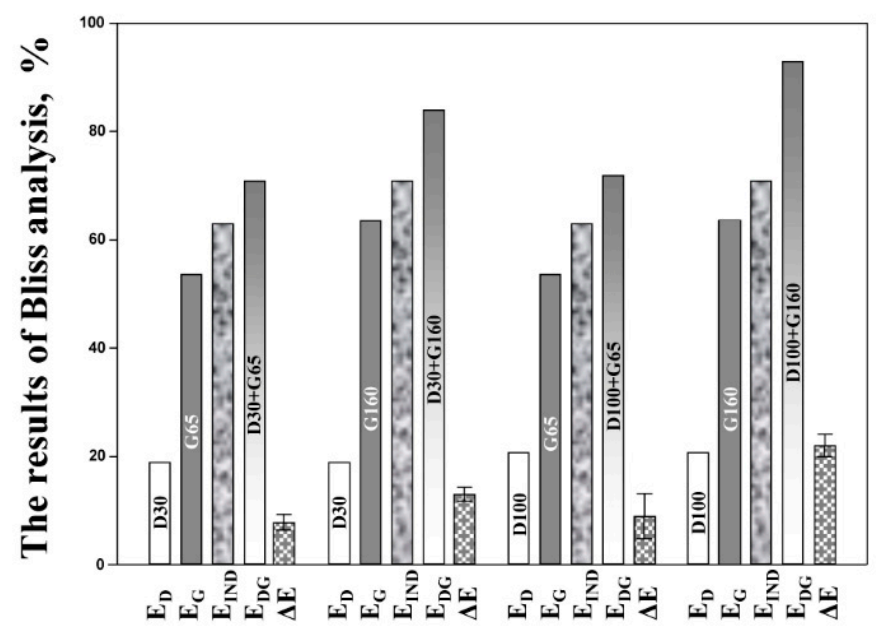

Figure 2. The results of Bliss independence drug interaction analysis of daptomycin and gentamicin combinations in an in vitro dynamic model. 


\section{4. $A B B C-A U C_{24} / M I C$ Relationships}

The enhancement of anti-staphylococcal efficiency of daptomycin and gentamicin combinations could be explained by increased $S$. aureus susceptibility to the antibacterials in the presence of each other (Table 1). In terms of $\mathrm{AUC}_{24} / \mathrm{MIC}$, the actual exposures of daptomycin given with gentamicin and gentamicin given with daptomycin are much greater than in single treatments. For example, daptomycin $\mathrm{AUC}_{24} / \mathrm{MICs}$ under the influence of gentamicin increased from $60 \mathrm{~h}$ (30/0.5, regimen D30) to $500 \mathrm{~h}(30 / 0.06 \mathrm{~h}$, regimen D30+G65) and $1000 \mathrm{~h}(30 / 0.03 \mathrm{~h}$, regimen D30+G160). Similarly, gentamicin $\mathrm{AUC}_{24} / \mathrm{MICs}$ under the influence of daptomycin increased from $260 \mathrm{~h}(65 / 0.25$, regimen $\mathrm{G} 65)$ to $380 \mathrm{~h}\left(65 / 0.17\right.$, regimen D100+G65) and $500 \mathrm{~h}$ (65/0.13, regimen D30+G65). Similar $\mathrm{AUC}_{24} / \mathrm{MIC}$ augmentations were obtained with other daptomycin plus gentamicin combinations (see Table 1).

To explore if the increase in the area between the control growth curve and each time-kill curve (ABBC) observed with the antibiotic combinations can be explained by these greater $\mathrm{AUC}_{24} / \mathrm{MIC}$ ratios, $\mathrm{ABBC}$ sere plotted against $\mathrm{AUC}_{24} / \mathrm{MICs}$ simulated in both single and combined antibiotic treatments (Figure 3). As seen in the figure, the $\mathrm{AUC}_{24} / \mathrm{MICs}$ of daptomycin (Figure 3a) and gentamicin (Figure 3b) used in combination were shifted to the right along the abscissa, i.e., to the area of higher $\mathrm{AUC}_{24} / \mathrm{MIC}$ values providing reasonable sigmoid relationships with $A B B C$.

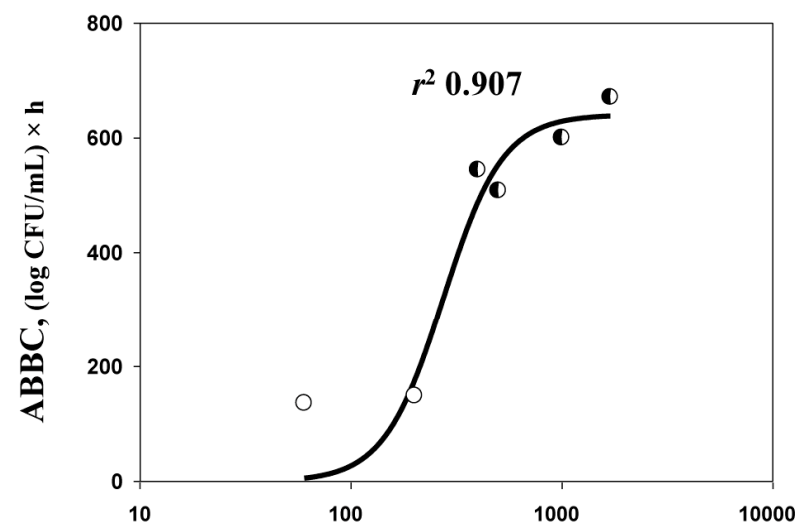

(a)

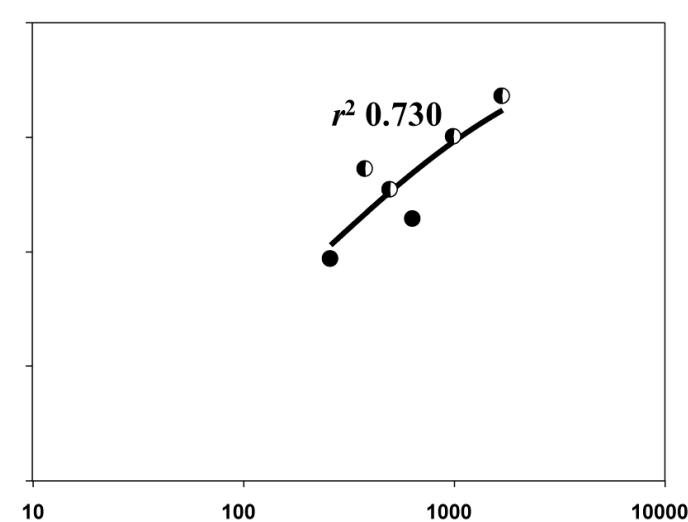

(b)

Daptomycin $\mathrm{AUC}_{24} / \mathrm{MIC}$, h

Figure 3. $\mathrm{AUC}_{24} / \mathrm{MIC}$-related areas between the control growth curve and each time-kill curve (ABBCs) observed with S. aureus 293 in single treatments with daptomycin (open circles) or gentamicin (filled circles) and in combined treatments (half-filled circles). The relationships fit by Equation (1): (a) $Y_{0}=0$, $x_{0}=2.438, a=640.7, b=0.1409$ and (b) $Y_{0}=0, x_{0}=2.385, a=804.0, b=0.5956$.

\section{Discussion}

Daptomycin plus gentamicin combinations were characterized primarily as indifferent by the results of FIC analyses ( $\sum$ FICs $0.64-1.18$ ); at only one concentration ratio with slight prevalence of gentamicin (1:1.5) did the antibiotic combination demonstrate enhanced anti-staphylococcal activity considered as synergistic ( $\sum$ FIC 0.48) (Table 1). More encouraging conclusions were drawn from 5-day dynamic model simulations where the anti-staphylococcal effects observed in each combined therapy (expressed as \% of the ABBC responsible for the maximal antibacterial effect) was greater than expected assuming the Bliss independence principle ( $\Delta E$ from 8 to $22 \%$ ), and statistically significant synergism was confirmed (Figure 2). The enhancement of antibacterial effects in combination therapy was more pronounced with the daptomycin-to-gentamicin $\mathrm{AUC}_{24}$ ratio 1:1.5 (regimen D100+G160) compared to other combined treatment regimens. In addition, only at regimen D100+G160 was bacterial regrowth fully prevented. Interestingly, the same antibiotic concentration ratio (1:1.5) was assessed as synergistic by the FIC analysis. In addition, the relatively higher $\sum F I C$ indices for other antibiotic combinations that predicted only indifferent interactions $(0.64-1.18)$ were consistent with the 
relatively smaller $\Delta E$ s determined for respective combined treatments (8-13\%) as compared with $\Delta E$ at $\mathrm{AUC}_{24}$ ratio 1:1.5 (22\%).

The results of FIC analyses are consistent with the pharmacodynamic data with respect to daptomycin and gentamicin interactions when the antibiotics were used in combination. It is highly likely that this observation is due to the use of the same antibiotic concentration ratios in both susceptibility testing and $\mathrm{AUC}_{24}$ ratios in the pharmacokinetic simulations. The predictive potential of susceptibility testing determined at pharmacokinetically derived concentration ratios was confirmed in our previous study with daptomycin and rifampin [17] where the $\sum F I C$ indices adequately reflected the ability of pharmacodynamic data to predict synergism or additivity.

Discrepancies between the results of synergy studies reported by other investigators with various antibiotic combinations including daptomycin plus gentamicin have been discussed [31-33]. These discrepancies were documented even when checkerboard and time-kill assays were performed in the same laboratory [20,34-36]. Most likely, this may have resulted from the use of arbitrarily chosen antibiotic concentration ratios that usually do not match each other in different methods. Results obtained using different ratios for checkerboard and time-kill assays may differ significantly. In light of our current data, the most effective concentration ratios obtained in checkerboard studies should be used in time-kill assays (including in dynamic models). From this viewpoint, when the results of these two methodologies with various daptomycin containing combinations including daptomycin plus gentamicin were compared against Staphylococci and Enterococci [20], consistent predicted interactions were achieved only at equal antibiotic concentration ratios.

Enhancement or improvement in daptomycin efficacy against daptomycin-susceptible S. aureus strains in the presence of gentamicin was described previously in studies with in vitro dynamic models [24-27]. However, advantages of combined therapy compared to mono-treatments were seen only in the first $24-36 \mathrm{~h}$ of observation as continued daptomycin alone provided sustained bactericidal activity up to the $72 \mathrm{~h} \mathrm{[24]} \mathrm{or} 96 \mathrm{~h}$ endpoint $[25,26]$. This is due most likely to the high therapeutic $\mathrm{AUC}_{24} \mathrm{~S}$ of daptomycin $(750-1750 \mathrm{mg} \cdot \mathrm{h} / \mathrm{L})$ that were responsible for the pronounced antibacterial effect. As a result, the relative portion of daptomycin combined with gentamicin in terms of the $\mathrm{AUC}_{24}$ ratio was quite prominent: daptomycin-to-gentamicin $\mathrm{AUC}_{24}$ ratios of 15-30:1. Comparative efficacy between regimens with and without gentamicin could not be distinguished over the duration of the experiment, making it difficult to adequately assess the actual synergistic potential of the combination regimens. The relative deficiency of gentamicin in the daptomycin combinations probably played a key role in the failure of the combination to improve daptomycin's weak efficacy against daptomycin-non-susceptible S. aureus strains [26]. The same considerations could apply to the results of an in vivo study in humanized rabbits where the addition of gentamicin did not enhance daptomycin efficacy due to a disproportional daptomycin-to-gentamicin $\mathrm{AUC}_{24}$ ratio $(850$ to $15 \mathrm{mg} \cdot \mathrm{h} / \mathrm{L}$, i.e., 60-fold difference) [23].

In the current study, the synergistic potential of daptomycin-gentamicin combinations was observed with the results of both checkerboard and pharmacodynamic experiments at comparable antibiotic concentrations or $\mathrm{AUC}_{24} \mathrm{~s}$, particularly with the use of subtherapeutic daptomycin exposures. Perhaps a way to improve the efficacy of daptomycin-gentamicin combinations is to use the antibacterials at doses that provide comparable $\mathrm{AUC}_{24} \mathrm{~s}$, usually representing lower daptomycin $\mathrm{AUC}_{24} \mathrm{~s}$. However, our results should be considered provisional and need additional confirmation in other experimental models. Of note, the $S$. aureus strains used in an experimental endocarditis model produced biofilms that could influence the pharmacodynamic interactions of daptomycin and gentamicin; this might have been responsible for the antagonism observed in that study [27].

In the current study, the potentiation of anti-staphylococcal effects of daptomycin-gentamicin combinations relative to monotherapy with the respective drugs was consistent with increased S. aureus susceptibility to these antibiotics in the presence of each other. This observation was confirmed by relating antibacterial effects of the combinations with respective antibiotic $\mathrm{AUC}_{24} / \mathrm{MIC}$ calculated by using MICs determined at pharmacokinetically derived concentration ratios (Figure 3). As seen in the 
figure, $\mathrm{ABBC}$ s determined in combined and single treatments could be plotted on the same sigmoid graph against $\mathrm{AUC}_{24} / \mathrm{MIC}$ of daptomycin (Figure 3a) or gentamicin (Figure 3b) with $r^{2} 0.907$ or 0.730 , respectively. As a result, reasonable $\mathrm{AUC}_{24} / \mathrm{MIC}-\mathrm{ABBC}$ relationships were demonstrated: the higher daptomycin or gentamicin $\mathrm{AUC}_{24} / \mathrm{MIC}$, the greater the bacterial killing.

Usual predictions of antibacterial effects of daptomycin-gentamicin combinations are based on $\mathrm{AUC}_{24} / \mathrm{MIC}$-dependent relationships for each drug separately and do not consider that the observed effect is provided by both drugs simultaneously. To more appropriately relate the antibacterial effect with total exposure of both drugs, the sum of simulated daptomycin and gentamicin $\mathrm{AUC}_{24} / \mathrm{MIC}$ ratios in combined treatments was calculated and noted as $\sum \mathrm{AUC}_{24} / \mathrm{MIC}$. Using pooled data, a sigmoid relationship between $\sum A \mathrm{AUC}_{24} / \mathrm{MIC}$ and $\mathrm{ABBC}$ was obtained (Figure 4 ). Despite finding that gentamicin alone produced a relatively low $\mathrm{AUC}_{24} / \mathrm{MIC}-\mathrm{ABBC}$ relationship $\left(r^{2} 0.730\right)$ compared to daptomycin $\left(r^{2}\right.$ 0.907; Figure 3), the $\sum \mathrm{AUC}_{24} / \mathrm{MIC}-\mathrm{ABBC}$ relationship for the combined data was described with a high correlation coefficient squared $\left(r^{2}\right.$ 0.915; Figure 4). Using a single relationship for the combined drugs is more appropriate since it considers cumulative exposures responsible for the total antibacterial effect.

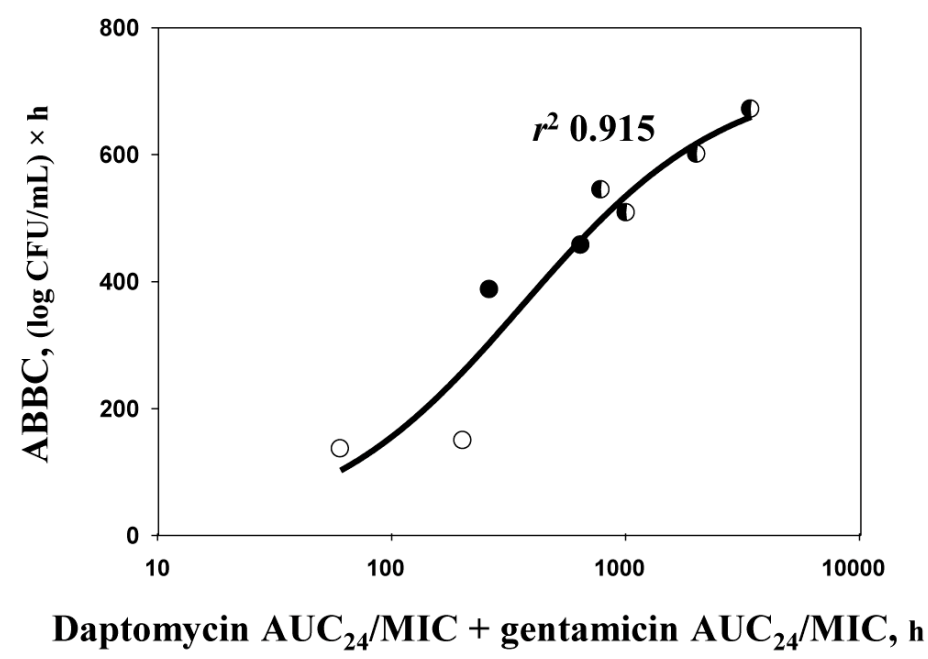

Figure 4. The sum of $\mathrm{AUC}_{24} / \mathrm{MICs}$ of daptomycin and gentamicin related to the $\mathrm{ABBC}$ observed with S. aureus 293 in single treatments with daptomycin (open circles) or gentamicin (filled circles) and in combined treatments (half-filled circles). The relationships fit by Equation (1): $Y_{0}=0, x_{0}=2.559$, $a=728.9, b=0.4316$.

The use of only one staphylococcal strain is a limitation of this study. Additional experiments are needed with more $S$. aureus strains exposed to daptomycin-gentamicin combinations as studied here. Along with that, relatively high limit of accurate detection of living bacteria in the system $\left(2 \times 10^{3} \mathrm{CFU} / \mathrm{mL}\right)$ throughout the observation period is another limitation of this study, as we could not see differences between the antibacterial effects of dosing regimens below this threshold. This could be important in determining the true antibacterial potential of the antibiotics (alone or in combination). Another limitation is that we did not consider any effect of protein binding (PB), which might influence antibacterial activity of the drugs. Unfortunately, the optimal method for properly correcting simulated profiles for PB is still undefined. Our detailed explanation of why we do not adjust the simulated antibiotic AUC/MICs for PB is described in a previous study with daptomycin [28]. 


\section{Materials and Methods}

\subsection{Antimicrobial Agents and Bacterial Strain}

Daptomycin powder was purchased from Acros Organics (Geel, Belgium); gentamicin sulfate was purchased from PhytoTechnology Laboratories (Shawnee Mission, KS, USA). Clinical isolate S. aureus 293 was used in the study.

\subsection{Antibiotic Dosing Regimens and Simulated Pharmacokinetic Profiles}

Simulated $\mathrm{AUC}_{24} \mathrm{~S}$ of daptomycin both in single and combined treatments corresponded to sub-therapeutic dosing regimens and were equal to 30 and $100 \mathrm{mg} \cdot \mathrm{h} / \mathrm{L}$ (single treatments were designated as D30 and D100, respectively). Gentamicin $\mathrm{AUC}_{24} \mathrm{~s}$ in mono and combined treatments corresponded to once daily doses $5 \mathrm{mg} / \mathrm{kg}$ and $7 \mathrm{mg} / \mathrm{kg}$. The respective $\mathrm{AUC}_{24} \mathrm{~s}, 65$ and $160 \mu \mathrm{g} \cdot \mathrm{h} / \mathrm{mL}$, used in pharmacokinetic simulations (single treatments were designated as G65 and G160, respectively), were calculated using peak serum gentamicin concentrations reported in human studies (16.6 and $39.8 \mu \mathrm{g} / \mathrm{mL}$, respectively, [37,38]) and $\mathrm{t}_{1 / 2}(3 \mathrm{~h},[38])$. The simulated combined treatments included: daptomycin $\mathrm{AUC}_{24} 30 \mathrm{mg} \cdot \mathrm{h} / \mathrm{L}+$ gentamicin $\mathrm{AUC}_{24} 65$ or $160 \mathrm{mg} \cdot \mathrm{h} / \mathrm{L}$ (regimens D30+G65 and D30+G160, respectively), daptomycin $\mathrm{AUC}_{24} 100 \mathrm{mg} \cdot \mathrm{h} / \mathrm{L}+$ gentamicin $\mathrm{AUC}_{24} 65$ or $160 \mathrm{mg} \cdot \mathrm{h} / \mathrm{L}$ (regimens D100+G65 and D100+G160).

With all dosing regimens, a series of monoexponential profiles that mimic once-daily dosing of daptomycin with a half-life of $9 \mathrm{~h}$ [39] and gentamicin with a half-life of $3 \mathrm{~h}$ alone or in combination were simulated for 5 consecutive days.

\subsection{In Vitro Dynamic Model}

A previously described dynamic model was used in simulations of single drug treatments with daptomycin and gentamicin [40]. Briefly, the model consists of two connected flasks, one containing fresh Mueller-Hinton broth supplemented with $50 \mathrm{mg}$ of $\mathrm{Ca}^{2+} / \mathrm{L}$ (CSMHB), because daptomycin antimicrobial activity is influenced by the presence of $\mathrm{Ca}^{2+}$ [41], and the other with a magnetic stirrer, the central unit, with the same broth containing either a bacterial culture alone (growth control experiment) or a bacterial culture plus antibiotic (killing/regrowth experiments). Peristaltic pumps circulated fresh nutrient medium to and from the central unit (volume $100 \mathrm{~mL}$ ) at a flow rate of $7.7 \mathrm{~mL} / \mathrm{h}$ for daptomycin or $23.2 \mathrm{~mL} / \mathrm{h}$ for gentamicin.

To simulate combination treatments, the model was modified according to the Blaser and Zinner principle to provide simultaneous mono-exponential elimination of daptomycin and gentamicin [42]. The model was supplemented with an additional $200 \mathrm{~mL}$ flask with fresh CSMHB containing daptomycin at initial concentrations equal to those in the central unit. Peristaltic pumps circulated fresh nutrient medium to and antibiotic-containing medium (both daptomycin and gentamicin) from the central unit at a flow rate of $23.2 \mathrm{~mL} / \mathrm{h}$ that corresponds to the antibiotic with the shorter half-life, i.e., gentamicin. To compensate for a too rapid decrease in concentrations of antibiotic with the longer half-life (daptomycin), peristaltic pumps circulated fresh medium to and daptomycin containing medium from the additional flask to the central unit at a flow rate of $15.5 \mathrm{~mL} / \mathrm{h}(23.2 \mathrm{~mL} / \mathrm{h}-7.7 \mathrm{~mL} / \mathrm{h})$.

The operation procedure used in the pharmacodynamic experiments was as described elsewhere [43]. Each experiment was performed at least in duplicate. Antibiotic dosing and sampling of the central unit were processed automatically, using computer-assisted controls. The system was filled with sterile CSMHB and placed in an incubator at $37^{\circ} \mathrm{C}$. The central unit was inoculated with an $18 \mathrm{~h}$ culture of $S$. aureus 293. After a $2 \mathrm{~h}$ incubation, the resulting exponentially growing cultures reached $\sim 10^{8}$ colony-forming units (CFU)/mL ( $10^{10} \mathrm{CFU}$ per $100 \mathrm{~mL}$ central unit). Then, antibiotics were administered into the central unit of the model. The duration of each experiment was $120 \mathrm{~h}$. 


\subsection{Susceptibility Testing}

Susceptibility testing was performed in triplicate using broth microdilution techniques according to Clinical and Laboratory Standards Institute (CLSI) methods [44]. After $24 \mathrm{~h}$ post-exposure with the organism grown in CSMHB, trays with twofold dilutions with known daptomycin and/or gentamicin concentrations were inoculated with $S$. aureus for a final concentration of approximately $5 \times 10^{5} \mathrm{CFU} / \mathrm{mL}$. The plates were incubated at $37^{\circ} \mathrm{C}$ for $18 \mathrm{~h}$.

With each antibiotic combination, stock solution concentration ratios of daptomycin to gentamicin corresponded to their $\mathrm{AUC}_{24}$ ratios used in the pharmacokinetic simulations and were as follows: 1.5:1 (regimen D100+G65), 1:1.5 (regimen D100+G160), 1:2 (regimen D30+G65) and 1:5 (D30+G160). The same concentration ratios were maintained in each subsequent dilution.

To determine the type of interaction between daptomycin and gentamicin in combination, the FIC for each antibiotic in each combination and $\Sigma F I C$ were determined as:

FIC of daptomycin $\left(\right.$ FIC $\left._{D}\right)=$ MIC of daptomycin in combination/MIC of daptomycin alone

FIC of gentamicin $\left(F I C_{G}\right)=$ MIC of gentamicin in combination/MIC of gentamicin alone

$$
\Sigma F I C=F I C_{D}+F I C_{G}
$$

Synergism was defined as a $\Sigma F I C$ index $\leq 0.5$, indifference was defined as a $\Sigma F I C$ index $>0.5$ but $\leq 4.0$, and antagonism was defined as a $\Sigma F I C$ index $>4.0$ [45].

\subsection{Quantitation of the Antimicrobial Effect and Its Relationships with $A U C_{24} / M I C$ Ratios}

In each experiment, bacteria-containing medium from the central unit of the model was sampled to determine bacterial concentrations throughout the observation period. Samples $(100 \mu \mathrm{L})$ were serially diluted as appropriate and $100 \mu \mathrm{L}$ was plated onto Mueller-Hinton agar plates, which were placed in an incubator at $37^{\circ} \mathrm{C}$ for $24 \mathrm{~h}$. The lower limit of accurate detection was $2 \times 10^{3} \mathrm{CFU} / \mathrm{mL}$ (equivalent to 20 colonies per plate).

Based on time-kill data, the area between the control growth curve and each time-kill curve (ABBC) [46] was determined from the beginning of treatment to $120 \mathrm{~h}$.

Daptomycin or gentamicin $\mathrm{AUC}_{24} / \mathrm{MIC}$ relationships with $\mathrm{ABBC}$ observed in single and combined antibiotic treatments (merged data) were fitted by the sigmoid function:

$$
Y=Y_{0}+a /\left\{1+\exp \left[-\left(x-x_{0}\right) / b\right]\right\}
$$

where $Y$ is $\mathrm{ABBC}, x$ is $\log \left(\mathrm{AUC}_{24} / \mathrm{MIC}\right) ; Y_{0}$ and $a$ are the minimal and maximal values of the $\mathrm{ABBC}$, respectively; $x_{0}$ is $x$ corresponding to $a / 2$; and $b$ is a parameter reflecting sigmoidicity.

All calculations were performed using SigmaPlot 12 software.

\subsection{Pharmacodynamic Bliss Independence-Based Drug Interaction Analysis}

The Bliss independence principle was used to analyze the daptomycin-gentamicin interaction, assuming that the drugs do not interact with each other when used in combination [47-49]. Bliss independence is described by the equation:

$$
E_{\mathrm{IND}}=E_{\mathrm{D}}+E_{\mathrm{G}}-E_{\mathrm{D}} \times E_{\mathrm{G}}
$$

where $E_{\mathrm{IND}}$ is the expected anti-staphylococcal effect of daptomycin-gentamicin combinations calculated using the effects of respective mono-treatments with daptomycin $\left(E_{\mathrm{D}}\right)$ and gentamicin $\left(E_{\mathrm{G}}\right)$.

The $E_{\mathrm{D}}, E_{\mathrm{G}}$ and antibacterial effects observed in combined treatments with daptomycin plus gentamicin $\left(E_{\mathrm{DG}}\right)$ were calculated by dividing the respective experimental $\mathrm{ABBC}$ to the $\mathrm{ABBC}$ responsive for maximal antibacterial effect (the area between the control growth curve and the line 
drawn at the lower limit of detection determined from 0 to $120 \mathrm{~h}$ ) and expressed in $\%$. The difference between the $E_{\mathrm{DG}}$ and $E_{\mathrm{IND}}(\Delta E)$ reflects the type of interaction between the daptomycin and gentamicin in each combination and is considered to be as follows: (i) Bliss synergy when $\Delta E$ and the lower limit of the $95 \%$ confidence interval (CI) $>0$, (ii) Bliss antagonism when $\Delta E$ and the upper limit of the $95 \%$ $\mathrm{CI}<0$, (iii) Bliss independence in any other case when the $95 \% \mathrm{CI}$ of $\Delta E$ includes 0 .

\section{Conclusions}

This study suggests that (1) combinations of daptomycin with gentamicin against $S$. aureus were synergistic according to Bliss independence-based analysis of pharmacodynamic experiments and also by the results of FIC analyses; (2) the enhanced antibacterial effects of the combinations relative to mono-treatments are likely due to the increased susceptibility of $S$. aureus both to daptomycin and gentamicin in the presence of each other; (3) the antibacterial effects of antibiotic combinations can be predicted by $\mathrm{AUC}_{24} / \mathrm{MICs}$ calculated with the use of MICs of each antibiotic determined at pharmacokinetically derived concentration ratios; and (4) $\sum \mathrm{AUC}_{24} / \mathrm{MIC}$ is a reliable predictor of antibacterial efficacy of antibiotic combinations as it considers the summed exposures of both drugs responsible for the total antibacterial effect.

Author Contributions: Conceptualization, A.A.F. and S.H.Z.; methodology, A.A.F., S.H.Z. and M.V.G.; software, Y.A.P.; validation, Y.A.P., M.V.G. and E.N.S.; formal analysis, Y.A.P. and M.V.G.; investigation, M.V.G. and E.N.S.; resources, A.A.F.; data curation, M.V.G., E.N.S. and Y.A.P.; writing-original draft preparation, M.V.G.; writing-review and editing, M.V.G.; E.N.S., Y.A.P. and S.H.Z.; visualization, Y.A.P.; supervision, A.A.F. and M.V.G.; project administration, A.A.F. and M.V.G.; funding acquisition, A.A.F. All authors have read and agreed to the published version of the manuscript.

Funding: This research was performed in Gause Institute of New Antibiotics and was funded by Russian Science Foundation (RSF), grant number 18-15-00433.

Conflicts of Interest: The authors declare no conflict of interest.

\section{References}

1. Mariani, P.G.; Sader, H.S.; Jones, R.N. Development of decreased susceptibility to daptomycin and vancomycin in a Staphylococcus aureus strain during prolonged therapy. J. Antimicrob. Chemother. 2006, 58, 481-483. [CrossRef]

2. Skiest, D.J. Treatment failure resulting from resistance of Staphylococcus aureus to daptomycin. J. Clin. Microbiol. 2006, 44, 655-656. [CrossRef]

3. Bennett, J.W.; Murray, C.K.; Holmes, R.L.; Patterson, J.E.; Jorgensen, J.H. Diminished vancomycin and daptomycin susceptibility during prolonged bacteremia with methicillin-resistant Staphylococcus aureus. Diagn. Microbiol. Infect. Dis. 2008, 60, 437-440. [CrossRef] [PubMed]

4. Sharma, M.; Riederer, K.; Chase, P.; Khatib, R. High rate of decreasing daptomycin susceptibility during the treatment of persistent Staphylococcus aureus bacteremia. Eur. J. Clin. Microbiol. Infect. Dis. 2008, 27, $433-437$. [CrossRef] [PubMed]

5. Lee, C.H.; Wang, M.C.; Huang, I.W.; Chen, F.J.; Lauderdale, T.L. Development of daptomycin non susceptibility with heterogeneous vancomycin-intermediate resistance and oxacillin susceptibility in methicillin resistant Staphylococcus aureus during high-dose daptomycin treatment. Antimicrob. Agents Chemother. 2010, 54, 4038-4040. [CrossRef]

6. Rose, W.E.; Schulz, L.T.; Andes, D.; Striker, R.; Berti, A.D.; Hutson, P.R.; Shukla, S.K. Addition of ceftaroline to daptomycin after emergence of daptomycin-nonsusceptible Staphylococcus aureus during therapy improves antibacterial activity. Antimicrob. Agents Chemother. 2012, 56, 5296-5302. [CrossRef]

7. Dortet, L.; Anguel, N.; Fortineau, N.; Richard, C.; Nordmann, P. In vivo acquired daptomycin resistance during treatment of methicillin-resistant Staphylococcus aureus endocarditis. Int. J. Infect. Dis. 2013, 17, e1076-e1077. [CrossRef] 
8. Sabat, A.J.; Tinelli, M.; Grundmann, H.; Akkerboom, V.; Monaco, M.; Del Grosso, M.; Errico, G.; Pantosti, A.; Friedrich, A.W. Daptomycin resistant Staphylococcus aureus clinical strain with novel non-synonymous mutations in the mprF and vraS genes: A new insight into daptomycin resistance. Front. Microbiol. 2018, 9, 2705-2714. [CrossRef] [PubMed]

9. Hayden, M.K.; Rezai, K.; Hayes, R.A.; Lolans, K.; Quinn, J.P.; Weinstein, R.A. Development of daptomycin resistance in vivo in methicillin-resistant Staphylococcus aureus. J. Clin. Microbiol. 2005, 43, 5285-5287. [CrossRef] [PubMed]

10. Vikram, H.R.; Havill, N.L.; Koeth, L.M.; Boyce, J.M. Clinical progression of methicillin-resistant Staphylococcus aureus vertebral osteomyelitis associated with reduced susceptibility to daptomycin. J. Clin. Microbiol. 2005, 43, 5384-5387. [CrossRef] [PubMed]

11. Murthy, M.H.; Olson, M.E.; Wickert, R.W.; Fey, P.D.; Jalali, Z. Daptomycin non-susceptible meticillin-resistant Staphylococcus aureus USA 300 isolate. J. Med. Microbiol. 2008, 57, 1036-1038. [CrossRef]

12. Sakoulas, G.; Rose, W.; Rybak, M.J.; Pillai, S.; Alder, J.; Moellering, R.C., Jr.; Eliopoulos, G.M. Evaluation of endocarditis caused by methicillin-susceptible Staphylococcus aureus developing nonsusceptibility to daptomycin. J. Clin. Microbiol. 2008, 46, 220-224. [CrossRef]

13. Cunha, B.A.; Pherez, F.M. Daptomycin resistance and treatment failure following vancomycin for methicillin-resistant Staphylococcus aureus (MRSA) mitral valve acute bacterial endocarditis (ABE). Eur. J. Clin. Microbiol. Infect. Dis. 2009, 28, 831-833. [CrossRef]

14. Tenover, F.C.; Sinner, S.W.; Segal, R.E.; Huang, V.; Alexandre, S.S.; McGowan, J.E., Jr.; Weinstein, M.P. Characterisation of a Staphylococcus aureus strain with progressive loss of susceptibility to vancomycin and daptomycin during therapy. Int. J. Antimicrob. Agents 2009, 33, 564-568. [CrossRef]

15. Van Hal, S.J.; Paterson, D.L.; Gosbell, I.B. Emergence of daptomycin resistance following vancomycin-unresponsive Staphylococcus aureus bacteraemia in a daptomycin-naïve patient-A review of the literature. Eur. J. Clin. Microbiol. Infect. Dis. 2011, 30, 603-610. [CrossRef]

16. Santimaleeworagun, W.; Changpradub, D.; Thunyaharn, S.; Hemapanpairoa, J. Optimizing the dosing regimens of daptomycin based on the susceptible dose-dependent breakpoint against vancomycin-resistant Enterococci infection. Antibiotics 2019, 8, 245. [CrossRef]

17. Golikova, M.V.; Strukova, E.N.; Portnoy, Y.A.; Zinner, S.H.; Firsov, A.A. Predicting the antistaphylococcal effects of daptomycin-rifampicin combinations in an in vitro dynamic model. J. Antibiot. 2020, 73, 101-107. [CrossRef]

18. Bollenbach, T. Antimicrobial interactions: Mechanisms and implications for drug discovery and resistance evolution. Curr. Opin. Microbiol. 2015, 27, 1-9. [CrossRef]

19. Aktas, G.; Derbentli, S. In vitro activity of daptomycin combinations with rifampicin, gentamicin, fosfomycin and fusidic acid against MRSA strains. J. Glob. Antimicrob. Resist. 2017, 10, 223-227. [CrossRef]

20. Snydman, D.R.; McDermott, L.A.; Jacobus, N.V. Evaluation of in vitro interaction of daptomycin with gentamicin or beta-lactam antibiotics against Staphylococcus aureus and enterococci by FIC index and timed-kill curves. J. Chemother. 2005, 17, 614-621. [CrossRef]

21. Lee, Y.C.; Chen, P.Y.; Wang, J.T.; Chang, S.C. A study on combination of daptomycin with selected antimicrobial agents: In vitro synergistic effect of MIC value of $1 \mathrm{mg} / \mathrm{L}$ against MRSA strains. BMC Pharm. Toxicol. 2019, 20, 25. [CrossRef] [PubMed]

22. Credito, K.; Lin, G.; Appelbaum, P.C. Activity of daptomycin alone and in combination with rifampin and gentamicin against Staphylococcus aureus assessed by time-kill methodology. Antimicrob. Agents Chemother. 2007, 51, 1504-1507. [CrossRef] [PubMed]

23. Miro, J.M.; García-de-la-Maria, C.; Armero, Y.; Soy, D.; Moreno, A.; del Río, A.; Almela, M.; Sarasa, M.; Mestres, C.A.; Gatell, J.M.; et al. Addition of gentamicin or rifampin does not enhance the effectiveness of daptomycin in treatment of experimental endocarditis due to methicillin-resistant Staphylococcus aureus. Antimicrob. Agents Chemother. 2009, 53, 4172-4177. [CrossRef]

24. LaPlante, K.L.; Rybak, M.J. Impact of high-inoculum Staphylococcus aureus on the activities of nafcillin, vancomycin, linezolid, and daptomycin, alone and in combination with gentamicin, in an in vitro pharmacodynamic model. Antimicrob. Agents Chemother. 2004, 48, 4665-4672. [CrossRef] [PubMed]

25. Tsuji, B.T.; Rybak, M.J. Short-course gentamicin in combination with daptomycin or vancomycin against Staphylococcus aureus in an in vitro pharmacodynamic model with simulated endocardial vegetations. Antimicrob. Agents Chemother. 2005, 49, 2735-2745. [CrossRef] [PubMed] 
26. Rose, W.E.; Leonard, S.N.; Rybak, M.J. Evaluation of daptomycin pharmacodynamics and resistance at various dosage regimens against Staphylococcus aureus isolates with reduced susceptibilities to daptomycin in an in vitro pharmacodynamic model with simulated endocardial vegetations. Antimicrob. Agents Chemother. 2008, 52, 3061-3067. [CrossRef]

27. LaPlante, K.L.; Woodmansee, S. Activities of daptomycin and vancomycin alone and in combination with rifampin and gentamicin against biofilm-forming methicillin-resistant Staphylococcus aureus isolates in an experimental model of endocarditis. Antimicrob. Agents Chemother. 2009, 53, 3880-3886. [CrossRef]

28. Firsov, A.A.; Smirnova, M.V.; Lubenko, I.Y.; Vostrov, S.N.; Portnoy, Y.A.; Zinner, S.H. Testing the mutant selection window hypothesis with Staphylococcus aureus exposed to daptomycin and vancomycin in an in vitro dynamic model. J. Antimicrob. Chemother. 2006, 58, 1185-1192. [CrossRef]

29. Tam, V.H.; Kabbara, S.; Vo, G.; Schilling, A.N.; Coyle, E.A. Comparative pharmacodynamics of gentamicin against Staphylococcus aureus and Pseudomonas aeruginosa. Antimicrob. Agents Chemother. 2006, 50, 2626-2631. [CrossRef]

30. Zinner, S.H.; Golikova, M.V.; Strukova, E.N.; Portnoy, Y.A.; Firsov, A.A. Predicting antibiotic combination effects on the selection of resistant Staphylococcus aureus: In vitro model studies with linezolid and gentamicin. Int. J. Antimicrob. Agents 2018, 52, 854-860. [CrossRef]

31. Steenbergen, J.N.; Mohr, J.F.; Thorne, G.M. Effects of daptomycin in combination with other antimicrobial agents: A review of in vitro and animal model studies. J. Antimicrob. Chemother. 2009, 64, 1130-1138. [CrossRef] [PubMed]

32. Nadrah, K.; Strle, F. Antibiotic combinations with daptomycin for treatment of Staphylococcus aureus infections. Chemother. Res. Pract. 2011, 619321. [CrossRef] [PubMed]

33. Dhand, A.; Sakoulas, G. Daptomycin in combination with other antibiotics for the treatment of complicated methicillin-resistant Staphylococcus aureus bacteremia. Clin. Ther. 2014, 36, 1303-1316. [CrossRef] [PubMed]

34. Kelesidis, T.; Humphries, R.; Ward, K.; Lewinski, M.A.; Yang, O.O. Combination therapy with daptomycin, linezolid, and rifampin as treatment option for MRSA meningitis and bacteremia. Diagn. Microbiol. Infect. Dis. 2011, 71, 286-290. [CrossRef] [PubMed]

35. Rose, W.E.; Berti, A.D.; Hatch, J.B.; Maki, D.G. Relationship of in vitro synergy and treatment outcome with daptomycin plus rifampin in patients with invasive methicillin-resistant Staphylococcus aureus infections. Antimicrob. Agents Chemother. 2013, 57, 3450-3452. [CrossRef] [PubMed]

36. Lai, C.-C.; Chen, C.-C.; Lu, Y.-C.; Lin, T.-P.; Chen, H.-J.; Su, B.-A.; Chao, C.-M.; Chuang, Y.-C.; Tang, H.-J. The potential role of sulbactam and cephalosporins plus daptomycin against daptomycin-nonsusceptible VISA and H-VISA isolates: An in vitro study. Antibiotics 2019, 8, 184. [CrossRef]

37. Cobussen, M.; Stassen, P.M.; Posthouwer, D.; van Tiel, F.H.; Savelkoul, P.H.M.; Havenith, T.; Haeseker, M.B. Improving peak concentrations of a single dose regimen of gentamicin in patients with sepsis in the emergency department. PLoS ONE 2019, 14, e0210012. [CrossRef]

38. Demczar, D.J.; Nafziger, A.N.; Bertino Jr, J.S. Pharmacokinetics of gentamicin at traditional versus high doses: Implications for once-daily aminoglycoside dosing. Antimicrob. Agents Chemother. 1997, 41, 1115-1119. [CrossRef]

39. Dvorchik, B.H.; Brazier, D.; DeBruin, M.F.; Arbeit, R.D. Daptomycin pharmacokinetics and safety following administration of escalating doses once daily to healthy subjects. Antimicrob. Agents Chemother. 2003, 47, 1318-1323. [CrossRef]

40. Firsov, A.A.; Shevchenko, A.A.; Vostrov, S.N.; Zinner, S.H. Inter- and intra-quinolone predictors of antimicrobial effect in an in vitro dynamic model: New insight into a widely used concept. Antimicrob. Agents Chemother. 1998, 42, 659-665. [CrossRef]

41. Fuchs, P.C.; Barry, A.L.; Brown, S.D. Daptomycin susceptibility tests: Interpretive criteria, quality control, and effect of calcium on in vitro tests. Diagn. Microbiol. Infect. Dis. 2000, 38, 51-58. [CrossRef]

42. Blaser, J.; Stone, B.B.; Zinner, S.H. Two compartment kinetic model with multiple artificial capillary units. J. Antimicrob. Chemother. 1985, 15, 131-137. [CrossRef] [PubMed]

43. Golikova, M.V.; Strukova, E.N.; Portnoy, Y.A.; Dovzhenko, S.A.; Kobrin, M.B.; Zinner, S.H.; Firsov, A.A. Predicting effects of antibiotic combinations using MICs determined at pharmacokinetically derived concentration ratios: In vitro model studies with linezolid- and rifampicin-exposed Staphylococcus aureus. J. Chemother. 2017, 29, 267-273. [CrossRef] [PubMed] 
44. Clinical and Laboratory Standards Institute. Performance Standards for Antimicrobial Susceptibility Testing: Twenty-Fifth Informational Supplement M100-S25; CLSI: Wayne, PA, USA, 2015.

45. Eliopoulos, G.M.; Moellering Jr, R.C. Laboratory methods used to assess the activity of antimicrobial combinations. In Antibiotics in Laboratory Medicine; Lorian, V., Ed.; The Williams \& Wilkins Co.: Baltimore, MD, USA, 1991; pp. 432-492.

46. Firsov, A.A.; Saverino, D.; Ruble, M.; Gilbert, D.; Manzano, B.; Medeiros, A.A.; Zinner, S.H. Predictors of effect of ampicillin-sulbactam against TEM-1 beta-lactamase-producing Escherichia coli in an in vitro dynamic model: Enzyme activity versus MIC. Antimicrob. Agents Chemother. 1996, 40, 734-738. [CrossRef]

47. Bliss, C.I. The toxicity of poisons applied jointly. Ann. Appl. Biol. 1939, 26, 585-615. [CrossRef]

48. Greco, W.R.; Bravo, G.; Parsons, J.C. The search for synergy: A critical review from a response surface perspective. Pharm. Rev. 1995, 47, 331-385.

49. Vakil, V.; Trappe, W. Drug combinations: Mathematical modeling and networking methods. Pharmaceutics 2019, 11, 208. [CrossRef]

(C) 2020 by the authors. Licensee MDPI, Basel, Switzerland. This article is an open access article distributed under the terms and conditions of the Creative Commons Attribution (CC BY) license (http://creativecommons.org/licenses/by/4.0/). 